\title{
Top Challenges in the Visualization of Engineering Tensor Fields
}

\author{
Mario Hlawitschka, Ingrid Hotz, Andrea Kratz, G.Elisabeta Marai, \\ Rodrigo Moreno, Gerik Scheuermann, Markus Stommel, Alexander Wiebel, \\ Eugene Zhang
}


ing tensors often involves solving multiple different technical problems at the same time - including visual intuitiveness, scalability, interactivity, providing both detail and context, integration with modeling and simulation, representing uncertainty and managing multi-fields; as well as overcoming terminology barriers and advancing research in the mathematical aspects of tensor field processing. We further note the need for tools and data repositories to encourage faster advances in the field. Our interest in creating and proposing this list is to initiate a discussion about important research issues within the visualization of engineering tensor fields.

\section{Introduction}

Many engineering disciplines make extensive use of tensors [1]. For example, applications in solid mechanics, civil engineering, bioimaging and bioengineering, computational fluid dynamics, geology and electrical engineering require the processing of tensor fields as part of domain-specific modeling, simulation, and analysis processes.

Given the complexities of engineering tensor data - including large scale visualization can be a powerful ally. In the last few years, this fact has fostered research in the visualization and processing of engineering tensor fields within the visualization community. The importance of these efforts relies on the huge potential impact of using advanced techniques of visualization in helping engineering professionals and scientists to have a better understanding of problems that involve processing of tensor fields. In addition, including features such as interactivity in the processing pipeline can be used to improve the results yielded by numerical simulations.

Visualization of engineering tensors fields is, however, a relatively new research topic $[11,3,4]$. Despite the potential advantages of tensor visualization in engineering, significant challenges make advances in the field difficult. Asking ourselves what the most important research challenges facing us are, and identifying the stumbling blocks, as well as the required practices, has the potential to speed up our progress. In this chapter, we attempt to start a discussion of these issues by proposing a list of top research challenges and issues in the visualization of engineering tensor fields.

We have been assembling this list of challenges over a series of discussions which included both visualization researchers and domain practitioners. Our interest in creating and proposing this list is not to impose our own ideas on the field, but rather to jump-start a discussion about important research issues [2] within the visualization of engineering tensor fields. We expect that this list will grow as the field itself grows and as additional topics are identified.

Here follows our list of the top challenges in the Visualization and Processing of Engineering Tensors. Please note that this list is not ranked. 


\section{Mathematical Models}

Tensors are perhaps one of the most commonly used concepts in physics, geometry, engineering, and medical research. There has been much research in tensors and tensor fields in terms of their mathematical and numerical analysis as well as geometric and physical properties. In the visualization community much groundwork has been carried out in the visualization of tensor fields. However, there are significant gaps between what we already know about tensors and what we can do with them, both in the visualization community and the application domains. This is due to a number of challenges that we face today.

First, mathematical analysis and visualization of tensor fields is intrinsically difficult, thanks to the large amount of information contained even in a single secondorder tensor (four numbers in 2D and nine in 3D). Existing analysis mainly focuses on local (e.g., pointwise) tensor properties, and relatively little work exists in global tensor field analysis, such as tensor field topology. Even in low-dimensional cases, typical tensor data can consist of millions of nodes, each of which is associated with a tensor. The sheer amount of data poses great challenges in robust analysis and effective visualization of tensor fields.

Second, mathematical analysis of tensor fields is further complicated by the order of tensors. The order of a tensor refers to the number of indices that is needed to describe the entries in the tensor. Zeroth-, first-, and second-order tensors refer to scalars, vectors, and matrices. While there is great need to handle tensors of order higher than two, our ability to process such tensors are rather limited. For example, spectral analysis for higher-order tensors is more difficult than that for second-order tensors, and it has not been found how to extend the notion of eigenvalues and eigenvectors to higher-order tensors that would satisfy all the properties that eigenvalues and eigenvectors of second-order tensor fields possess. Moreover, global structures in higher-order tensors are not well understood, and it is not yet clear what information is essential for understanding higher-order tensors. In addition, higher-order tensors contain more entries, which makes the storage and effective processing more challenging than their lower-order counterparts such as vectors and matrices.

\section{Relevant Quantities and Terminology}

In many application areas, e.g., solid mechanics, the use of tensor related quantities has a long tradition. In these areas a very application specific terminology has been developed. Thus often a multiple of identifiers or names exist for the same mathematical entity. This is already the case for basic entities such as eigenvalues: in the context of stress tensors in engineering eigenvalues are usually called principal stresses. Even more confusing, there are terms used across disciplines which have different definitions, e.g. anisotropy.

A good visualization is guided by the practical questions and relevant quantities of the application. Even more than in other visualization areas, the questions 
related to tensor fields strongly vary across application fields. Similar to the terminology, the relevant quantities cannot easily be transferred. The physical meaning of the mathematical properties deviates strongly. For example, while in some applications isotropic or degenerate points (points where the eigenvectors are not uniquely defined) are of special importance, in other contexts they are just points of high symmetry without particular meaning. We note that the terminology of tensor fields in mathematics, the physical (or application-dependent) interpretation of tensor fields in application areas, and the teminology from the application areas are all correct, from a reasonable perspective. However, clear translations of the various definitions across fields are needed.

In summary, it is difficult to transfer visualization methods from one application to another without specific adaptation. Entering a new application area always requires significant effort to get to know the domain-specific language, which can be discouraging to visualization researchers. Finally, accessing the relevant information is often not trivial since it is scattered in the literature and rarely do any concise introductions $[10,9]$ exist.

\section{Effective Visual Abstractions}

Effective visual abstractions are a fundamental problem in the visualization of engineering tensors. By effective we denote visual abstractions which capture the physical or mathematical aspects of the tensors and which are intuitive to the applicationdomain practitioners.

The visual abstraction issue is particularly difficult because the physical meaning of engineering tensors is not necessarily intuitive. Symmetric second order tensors are used routinely as abstract quantities in the mathematical modeling of turbulent combustion and considered very useful for computation. Yet only abstractions of the tensor, such as the trace of the tensor matrix, may bear physical meaning to the domain practitioner. In this context, it is important to be able to select from the many visual abstractions that have already been proposed, and to effectively combine them into frameworks that solve specific engineering problems.

The physical meaning of tensors can further greatly impact how they should be analyzed and visualized, even when the mathematical representations of these tensors are the same. Examples of this include the stress tensor and strain tensor from solid mechanics, the rateofdeformation tensor from fluid dynamics, and the diffusion tensor from medical imaging, all of which are secondorder, symmetric tensors. Yet, different mathematical analyses and visualizations are needed that best suit the domain scientists needs. While certain tensor visual abstractions have been established in other application domains, they do not necessarily transfer well to engineering. For example, unlike diffusion tensors in medical imaging, the eigenvectors and eigenvalues of engineering tensors may be meaningless in turbulent combustion; while reduction of the tensor field to a scalar field - e.g., stress in mechanical engineering, or divergence in turbulent combustion - may bear particularly intu- 

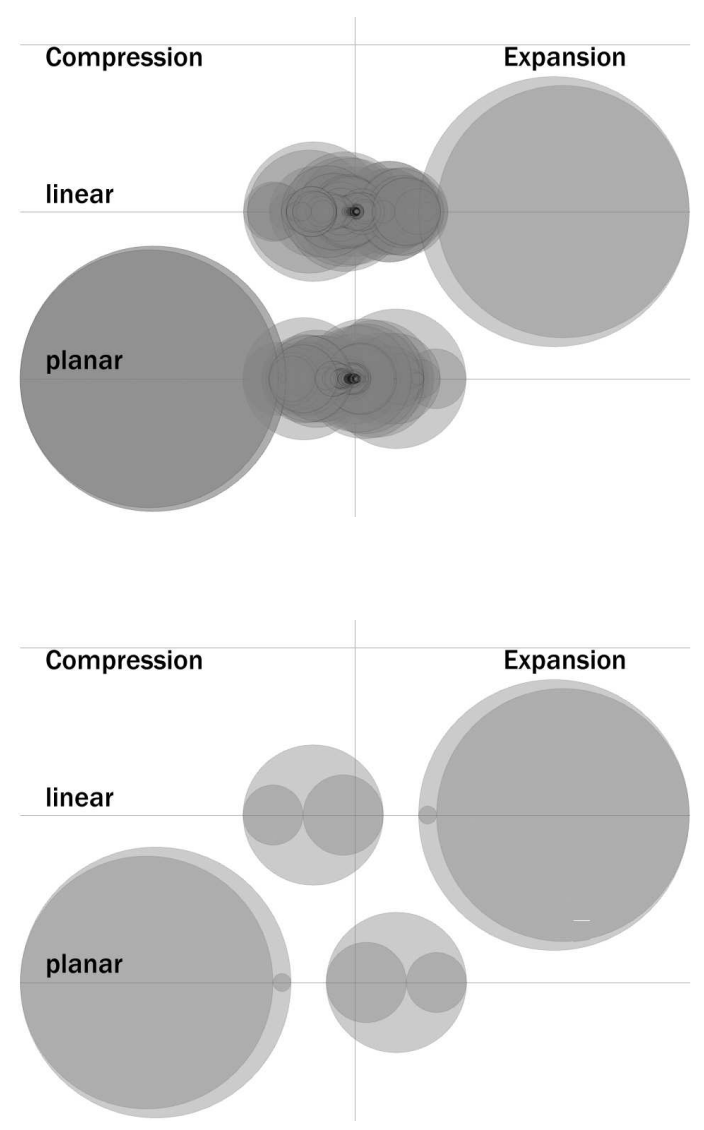

Fig. 1 Mohr diagram of a two-force dataset (simulation of a block on which a pushing and a pulling force are applied); top: unfiltered Mohr diagram, bottom: filtered Mohr diagram. The filtered Mohr diagram depicts only circles for cluster representatives that have been computed using mean-shift clustering in shape space. This representation reveals that four characteristic stresses occur: Compressive, tensile and mixed stresses. These characteristics are found in many engineering datasets.

itive meaning to the engineering practitioner. To create effective visual representations, visualization researchers need to spend significant time understanding the underlying science and engineering.

Finally, the particular visual abstraction depends on which aspect of the model are considered important. Finding effective, understandable visual abstractions for engineering tensors is thus a fundamentally interdisciplinary, exploratory process. Domain hypotheses and data change iteratively with the visual exploration process, thus the data models and thus visual abstractions change iteratively as well. Communication with the domain experts is needed to establish which aspects of the model are important and need to be captured by the visual representations. 


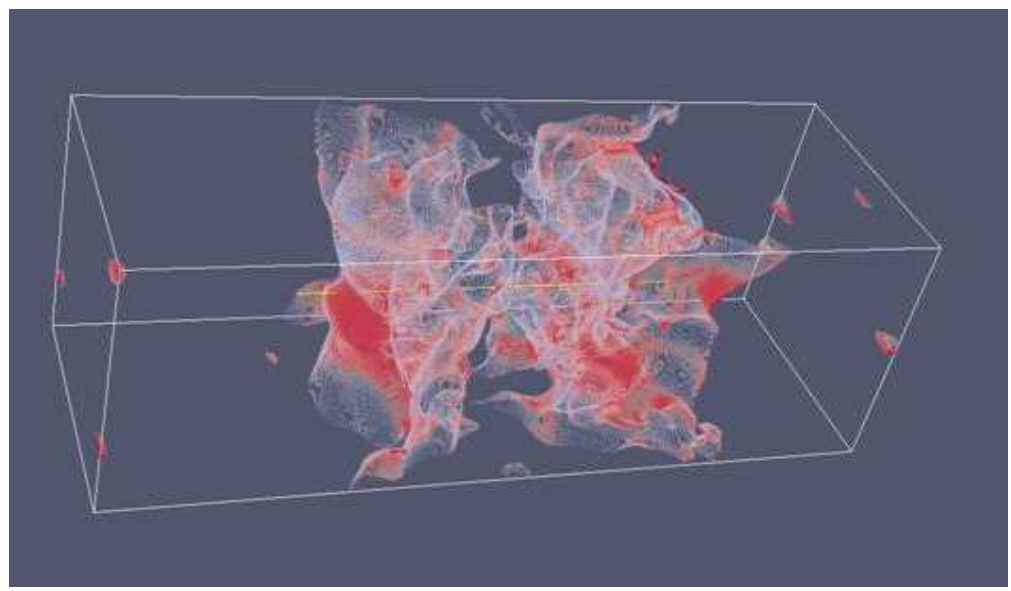

Fig. 2 Paraview rendering of the shock regions (points within 0.01 of the local speed/speed of sound ratio of 1) in a rich and very large $-21 \mathrm{M}$-points - combustion dataset. Due to the supersonic nature of this test case, the flow field exhibits shocklets during the simulation.

To identify the right or interesting quantities to visualize and to establish a common visual language with the domain specialists, visualization researchers have found it useful to cover the domain literature and seek traditional visual representations (Figure 1) [5]. Another approach is to tap into information visualization abstractions such as icons or glyphs through a parallel prototyping [7] approach. However, identifying the right invariants and visualization rule-of-thumbs for a particular domain — and then across engineering fields — remains a major challenge.

\section{Scalability: Very Large Datasets}

Engineering tensor data tend to be generated from computational simulations. Major advances in computing capabilities mean that recent datasets tend to have very large scale - petascale and exascale: even toy examples from turbulent combustion have millions of grid points (Figure 2).

Given the scale of these tensor fields, major scalability challenges include occlusion and clutter. For example, information encoded using glyph-representations becomes quickly unreadable. Furthermore, slow interaction with complex, though expressive geometric representations means hardware-accelerated techniques are needed to render and explore the data at interactive rates.

Possible solutions to scalability issues include abstraction (volume rendering [6, 5], Figure 3), interactive filtering (also projection to planes), clustering (also dimension reduction), or simply creating novel scalable visual representations. It may also be useful to borrow scalable ideas from machine learning and information visualization. 

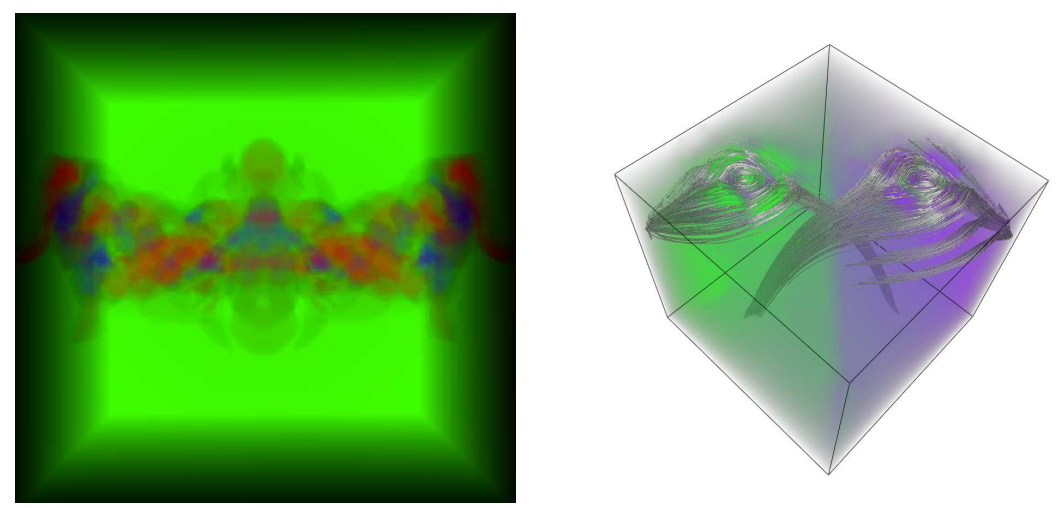

Fig. 3 Left: Volume rendering of a three tensor clustering for a 8M point mixing-layer combustion dataset (clusters mapped to green, red, and blue). Right: Hybrid Rendering of the two-force dataset. Volume rendering provides context (violet=tension, green=compression) and tensor lines are seeded for the pushing and pulling force only (along major and minor eigenvector, respectively).

\section{Scalability: Multi-field Datasets}

In addition to large scale, engineering data also typically feature multiple fields, including non-tensor quantities such as pressure, temperature, or velocity. For example, mechanical engineering problems may feature more than 60 tensor and nontensor field quantities in the same dataset [8]. Furthermore, numerical simulations may run over many time steps, in which case comparison of tensor fields across time becomes particularly important. We should be able to effectively visualize multiple fields simultaneously, and to visualize the interaction between multiple fields.

However, abstractions which meet successfully the large data scalability challenge - such as transfer-function based volume rendering or hybrid representations - do not necessarily facilitate the visual comparison of multiple fields at the same time. Established techniques for visual comparison - such as juxtaposition, overlays or animation - may require novel visual abstractions to meet the challenges of tensor multi-field data that stem from engineering fields.

\section{Scalability: Details in Context}

The shear spatial size combined with many small-scale phenomena provides additional challenges: Whereas details-in-context techniques have a long history in medical visualization and in information visualization, a transfer of those techniques to engineering data is typically not a trivial task that is made even harder due to the lack of dataset independent spatial reference other than the provided geometry. 

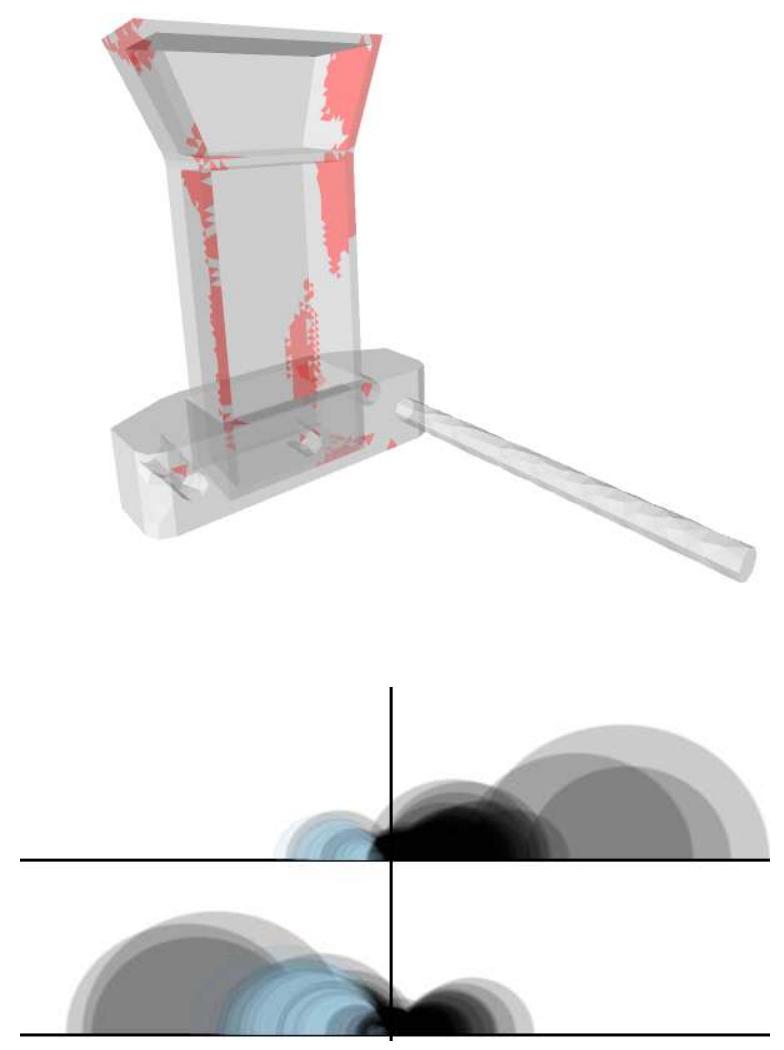

Fig. 4 Construction element dataset used for modeling and simulation in mechanical engineering. Visualization should be integrated with the modeling and simulation process, and not a postprocessing step. Top: Compressive areas are highlighted. Bottom: Mohr diagram. The dataset exhibits the same basic characteristics as the two-force dataset.

The delivery of visualization systems that follow basic interaction paradigms such as providing focus and context in tensor fields at the same time often rely on the possibility to display data at different levels of detail, and pan and zoom techniques require relatively smooth transitions between representations at different scales. Besides modified seeding strategies for glyphs and topology simplification, only few techniques currently used in tensor visualization fulfill these requirements and, to the best of our knowledge, none of these has been studied in detail in this context. 


\section{Integration of Visualization with Simulation and Modeling}

Most engineering problems do not require mere post-processing visualization, but interactive visual computing (Figure 4). For example, tensor visualization is fundamentally interesting for the validation or debugging of numerical simulations in the context of computational turbulent combustion modeling. In such situations, the domain experts are often interested in visually exploring tensor datasets as they are being generated by the numerical method used in the simulation. Detecting anomalies in the tensor field may highlight bugs in the modeling stage or in the numerical simulation stage. Early detection of such anomalies may help stopping in such cases lengthy, computationally expensive simulations that would lead to erroneous final results.

With simulation runtimes on the order of days or months even on supercomputers, the integration of visual analysis with the simulation and modeling processes could lead to significant benefits. Furthermore, the domain experts are interested in steering calculations in real-time, being able to change parameters on the fly and see the effects.

However, the scale of these simulations and the hardware on which they are run pose stringent constraints. Massively parallel simulations are common: How do we combine the results from such parallel simulations in an interactive rendering of the entire dataset? Furthermore, large scale datasets often cannot be saved to disk: How do we visualize tensor data when the domain expert cannot afford to stop the simulation to save the data for visualization? In situ visualization and remote visualization of tensor fields are, in this context, topics of high interest.

\section{Interpolation and Smoothing}

Tensor field data can come from direct measurement or numerical simulation. In both cases the data is only available at discrete locations (e.g., the vertices of a grid or integration points of a cell). However, most tensor field analysis and visualization approaches assume an everywhere continuous tensor field. Consequently, it is necessary to recontruct the tensor data from the vertices to the rest of the domain (e.g., points on the edges, faces, and interiors of grid cells). This process, referred to as interpolation or extrapolation, requires great care. While it seems simple and is rather straightforward to perform numerically, factors such as the size of the grid and its configuration as well as the used interpolation scheme are often ignored even though they impact the amount of faithfulness of the interpolated data with respect to the ground truth. However, without this being properly understood, errors may be introduced in the data that compromise the quality of the subsequent physical interpretation. We wish to point out that the difference between the ground truth and the interpolated tensor fields is difficult to quantify. It is similar to object reconstruction from photos, where the ground truth is generally not known, and therefore the difference between it and the reconstructed object is not available. Moreover, it is not 
clear how to reduce this difference. Consequently, the difference can be viewed as a form of error, or, as we argue here, a form of uncertainty.

Another useful and often necessary data processing operation is tensor field smoothing. This operation is designed to remove highfrequency signals from the data, under the assumption that such signals are noise and therefore of little relevance to the physical interpretation. However, such interpretation has not been validated for tensor field smoothing by the visualization community. It is not clear how the global structures (topological) in the tensor fields are impacted by the tensor field smoothing. In addition, there has not been a principled way of deciding how much smoothing is needed. Consequently, uncertainty is introduced into the data.

Another challenge is which mathematical representation for tensors should be used during smoothing. A popular approach is to perform tensor field smoothing on the entries of the tensors. While this seems to work well in practice in many applications, it is nonetheless often unjustified. Other approaches, such as smoothing on the norm, the eigenvalues and the eigenvectors of the tensor, can and should be explored and compared with smoothing on the tensor entries.

\section{Uncertain Data}

The fact that data is often given in a discrete form and the resulting need for interpolation is only one source of error found in simulated and measured data. As neither simulations nor measurements can be taken as ground truth, the introduced error can be handled as one kind of uncertainty in the data. In addition, a variety of errors accumulate in a data processing pipeline, most of which can be described roughly at the place of occurrence: i.e., the distortion of the measurement, measurement bias, discretization and quantification errors, calculation errors in pre-processing or simulation due to the limited mathematical, and, last but not least, the graphical precision of the screen.

Data processing pipelines, including visualization systems, would greatly profit from propagating errors from the step where they occur to the visualization and incorporating these errors in the visualization; whereas to date, the most-common approach is to keep every single error small and then ignore them. Error bars are the most common representation in all scientific journals but equivalent metaphors for the visualization of tensor data are rare.

The uncertainty of input data has further implications to the processing when combined with interpolation. Where interpolation in certain data leads to errors (or uncertainties) between the sample points, interpolation (seen as averaging) of uncertain data may actually reduce uncertainty. Whereas such models are being explored for scalar data, an application to tensor-valued data remains an open question and one of the most important challenges for the next years. 


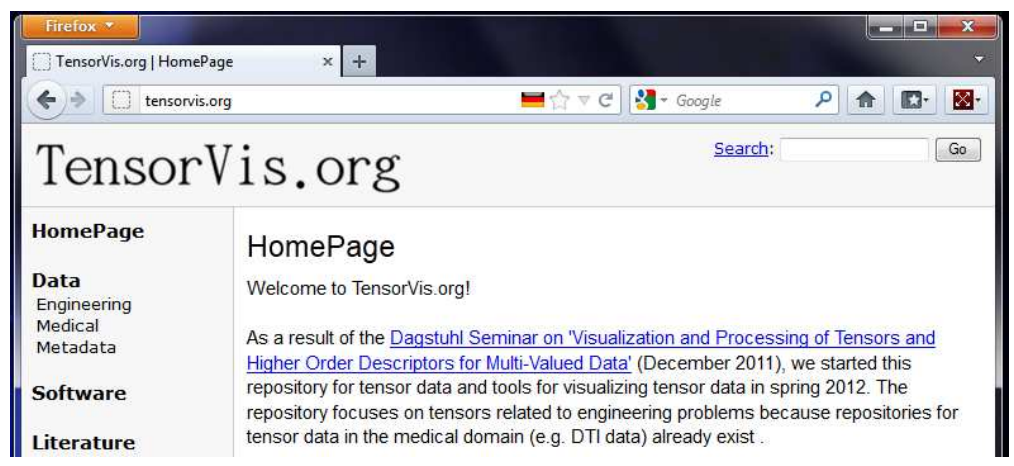

Fig. 5 Screenshot of the tensorvis.org website that hosts the prospective data and tool repositories.

\section{Data Repositories}

Progress in many scientific disciplines benefits from the availability of free data or benchmarks. This is especially true for visualization where data is the starting point for all techniques. The impact of such data is threefold: The data can be used to develop and test new techniques. It can help to show new research directions and demonstrate shortcomings of existing methods. Finally, it can be used to evaluate the applicability of new techniques, as well as their accuracy and efficiency compared to existing ones.

Looking at visualization research, freely available data did accelerate the developments at least in volume visualization (http://www.volvis.org, and others) and flow visualization (Vis-Contests 2004, 2006, 2011, and the International CFD Database at http://cfd.cineca.it/). Although not in one repository like volvis.org, for tensor visualization there exist a large number of freely available data sets from the medical domain (e.g. Kindlmann's data page, http://www.grand-challenge.org and Vis-Contest 2010) and some datasets related to earthquakes. However, scanning through the tensor visualization literature shows that there are only very few and, maybe more importantly, very simple datasets from engineering applications available. As the notion and use of tensors is very different in different application domains, this is a severe problem for the development of visualization techniques for such data. As a first step to mitigate this problem, we set up a data repository at http://www.tensorvis.org (Figure 5) in Spring 2012 and work towards populating it with various tensor datasets from engineering applications and meta information about these datasets. Maintaining minimum meta information requirements we hope to make the provided data even more useful. 


\section{Tool Repositories}

In addition to the availability of data, research can also be supported and even accelerated by the availability of software tools. Again, the impact is three-fold: Tools can help to convert, load and process the data to be examined, tools providing visualizations themselves can be used as benchmarks for new techniques, and, finally, researchers can integrate some tools or libraries into their own software.

Domain practitioners new to the field of tensor visualization are often unaware of the various existing tensor visualization tools and tensor processing libraries. A repository providing descriptions and web-links for download or further information would make their first steps in the field easier. Such repositories should be integrated with data repositories to have all relevant information in one place.

\section{Conclusion}

Analyzing the collection of challenges we introduced in this chapter, we note that creating visualization tools for engineering tensors often involves solving multiple different technical problems at the same time - including visual intuitiveness, scalability, interactivity, providing both detail and context, integration with modeling and simulation, representing uncertainty and managing multi-fields. This level of complexity usually leads to trade-offs among different strategies that aim at reasonably tackling all technical problems in the same framework. In addition, many mathematical aspects of tensor field processing, which are also necessary for visualization, are still under active research.

We further note that the gap between the availability of visualization tools and their actual use by domain practitioners on a regular basis is still huge. Although designing application-dependent visual abstractions is one of the strategies that can help to reduce such a gap, this usually requires visualization researchers to have a very good knowledge of the specific application, which is time consuming and not always effective. Finally, as in many other research areas, the lack of tools for sharing knowledge within the community has hitherto discouraged faster advances in the field.

In this chapter, we aimed to summarize the challenges in creating successful visualization tools for tensor fields in engineering. With this analysis and formalization of our collective experiences in the visualization of engineering tensors, we hope to motivate visualization researchers to think either about new tensor-related problems or about persistent tensor problems across engineering fields with a refreshed perspective.

Acknowledgements Grateful acknowledgments to H. Hagen (Kaiserslautern University), S. Levent Yilmaz, Mehdi Nik, Tim Luciani, Adrian Maries and Md. Abedul Haque (Pitt) for gracefully providing several of the images and captions in this chapter, as well as for inspiring discussions. G.E. Marai's work is partially supported through NSF IIS-0952720. 


\section{References}

1. T. Delmarcelle and L. Hesselink (1994) The Topology of Symmetric, Second-Order Tensor Fields, IEEE Visualization 1994, 140-147.

2. C.R. Johnson (2004) Top Scientific Visualization Research Problems. IEEE Computer Graphics and Applications, vol. 24, no. 4, pp. 13-17.

3. C. Dick, J. Georgii, R. Burgkart, and R. Westermann (2009) Stress Tensor Field Visualization for Implant Planning in Orthopedics. IEEE Transactions on Visualization and Computer Graphics 15, 6, 1399-1406.

4. V. Slavin, R. Pelcovits, G. Loriot, A. Callan-Jones, and D. Laidlaw (2006) Techniques for the Visualization of Topological Defect Behavior in Nematic Liquid Crystals. IEEE Transactions on Visualization and Computer Graphics 12, 5, 1323-1328.

5. A. Kratz, B. Meyer, I. Hotz (2010), A Visual Approach to Analysis of Stress Tensor Fields, ZIB-Report Technical Report 10-26.

6. A. Maries, Md. A. Haque, S. L. Yilmaz, M. B. Nik, G. E. Marai (2011). Interactive Exploration of Stress Tensors Used in Computational Turbulent Combustion. Springer-Verlag, New Developments in the Visualization and Processing of Tensor Fields, ed. D. Laidlaw and A. Villanova.

7. S. Dow, A. Glassco, J. Kass, M. Schwarz, D.L Schwarz (2010), S.R. Klemmer, Parallel Prototyping Leads to Better Design Results, More Divergence, and Increased Self-Efficacy, Transactions on Computer-Human Interaction, 11(4).

8. N.N.: Abaqus Analysis User's Manual, Version 6.7 EF, Providence, RI, USA. Dassault Systmes, Simulia Corp.

9. R. Brannon, Mohrs circle and more circles (2003), http://www.mech.utah.edu/ brannon/public/Mohrs Circle.pdf.[Online]. Available: http://www.mech.utah.edu/brannon/public/MohrsCircle.pdf

10. J. C. Kolecki, An Introduction to Tensors for Students of Physics and Engineering, NASA/TM2002-211716. [Online]. Available: http://www.grc.nasa.gov/WWW/k12/Numbers/Math/documents/Tensors_TM2002211716.pdf

11. Y. M. A. Hashash, J. I.-C. Yao, and D. C. Wotring (2003), Glyph and hyperstreamline representation of stress and strain tensors and material constitutive response, International Journal for Numerical and Analytical Methods in Geomechanics, vol. 27, no. 7, pp. 603626. 